\title{
Association of polycystic ovarian syndrome and metabolic syndrome in Tripura, India
}

\author{
Ipsita Batra, Jahar Lal Baidya*, Mamata Pradhan
}

Department of Obstetrics and Gynecology, Agartala Government Medical College, Agartala, Tripura, India

Received: 25 March 2020

Accepted: 22 April 2020

\section{*Correspondence:}

Dr. Jahar Lal Baidya,

E-mail: iamjlbaidya@hotmail.com

Copyright: () the author(s), publisher and licensee Medip Academy. This is an open-access article distributed under the terms of the Creative Commons Attribution Non-Commercial License, which permits unrestricted non-commercial use, distribution, and reproduction in any medium, provided the original work is properly cited.

\section{ABSTRACT}

Background: A total $4-11 \%$ of women in India are affected by PCOS. Studies show that incidence and prevalence is increasing. It is a constellation of metabolic and endocrine abnormalities with significant cost to quality and quantity of life. Aim of this study was to find out the association of metabolic syndrome with PCOS. Objectives of this study were identified subjects suffering from PCOS and measure strength of association of metabolic syndrome.

Methods: Case-control study conducted in the department of Obstetrics and Gynaecology Agartala Government Medical College, Agartala, Tripura from January 2017 to June 2018. Fifty cases were diagnosed by Rotterdam criteria and 50 cases age matched controls were recruited. Hundred subjects underwent evaluation for metabolic syndrome according to ESHRE/ASRM criteria. Statistical analysis was done using SPSS 17.0.

Results: Mean age was $19.4 \pm 3.5$ years. Oligomenorrhea found in 42 out of 50 cases. Hirsutism found in $54 \%$ of cases. There is a statistically significant difference in weight, BMI and waist circumference among PCOS cases and controls. Hypertriglyceridemia was found to be significantly higher among PCOS cases. Fasting glucose and 2-hour OGTT were found to be statistically significantly different. No association was found between metabolic syndrome and clinical hyperandrogenism, but metabolic syndrome was significantly associated with higher BMI. BMI, Hip circumference and oral glucose tolerance test were independent predictors of polycystic ovarian syndrome. Metabolic syndrome was present in $34 \%$ of cases. The odds ratio of having metabolic syndrome in a case of PCOS is 5.92.

Conclusions: Association between PCOS and metabolic syndrome is statistically significant. The two entities are intrinsically linked to each other and early identification of one may lead to the diagnosis and management of the other.

Keywords: Cardio metabolic syndrome, Clinical hyperandrogenism, European society of human reproduction and embryology guidelines, Metabolic syndrome, Polycystic ovarian syndrome

\section{INTRODUCTION}

Polycystic ovarian syndrome (PCOS) is one of the commonest endocrine disorders, affecting around 4-7\% of women of reproductive-age worldwide and 4 to $11 \%$ in India. ${ }^{1,2}$ It is now recognized as a common, heterogeneous, heritable disorder affecting women throughout their lifetime. PCOS is characterized by hyperandrogenism, ovulatory dysfunction and polycystic ovaries. However, there is considerable inter-individual variation in presentation. PCOS is not only a reproductive endocrinopathy but also a metabolic disorder.

PCOS is associated with hyperinsulinemia, glucose intolerance, obesity and altered lipid profile. Insulin resistance is thought to be the uniting pathogenic factor between hypertension, glucose intolerance, obesity, lipid abnormalities and coronary artery disease, which together constitutes metabolic syndrome or syndrome $\mathrm{X}^{3}$ Numerous studies worldwide have linked PCOS to 
diabetes mellitus type 2, obesity, dyslipidaemia but no such study was found to have been done in the state of Tripura.

Factors like ethnicity also contribute to variation in the association between metabolic derangements and PCOS in different population groups in different parts of the world. PCOS is no longer a rare gynaecological diagnosis but an endocrinopathy with multi-system sequelae. In this era of growing metabolic disturbances affecting the health of society, PCOS is an emerging area of utmost concern and therefore this study was undertaken.

In this study, presence of metabolic abnormalities is compared between PCOS cases and controls. Purpose of present work is to find out association of metabolic syndrome with polycystic ovarian syndrome among women attending out-patient department of Obstetrics and Gynaecology at Agartala Government Medical College, Agartala, Tripura, a tertiary care government hospital catering to patients from every corner of this state in the North-eastern zone of India, with population of various ethnic groups.

\section{METHODS}

Study population: All the patients, aged 15-45 years who attended the gynecology OPD of Agartala Govt. Medical College Hospital, Tripura during the study period (January 2016 to June 2017).

\section{Inclusion criteria}

- Non-pregnant females between ages 15-45 years

- Satisfying Rotterdam criteria, 2003 for PCOS. ${ }^{4}$

\section{Exclusion criteria}

- Abnormal prolactin level

- Abnormal Thyroid level.

\section{Diagnosis of metabolic syndrome}

Metabolic syndrome was diagnosed using the Rotterdam European society human reproduction and embryology/American society reproductive medicinesponsored (ESHRE/ASRM) PCOS consensus workshop group criteria. ${ }^{4}$

\section{Presence of three of the following criteria confirmed the diagnosis}

- $\quad$ Female waist $>35$ inches (more than $88 \mathrm{cms}$ )

- Triglycerides $>150 \mathrm{mg} / \mathrm{dL}$

- $\mathrm{HDL}<50 \mathrm{mg} / \mathrm{dL}$

- $\quad$ Blood pressure $>130 / 85 \mathrm{mmHg}$

- Fasting glucose: 110-126 mg/dL and/or two-hour glucose (75 gm OGTT): 140-199 mg/dL.

\section{Procedure}

Thorough history and physical examination were done after recruitment. Signs of clinical hyperandrogenism along with scoring of hirsutism were done using Ferriman Gallwey Score. ${ }^{5}$ Subjects then underwent ultrasonography for signs of polycystic ovaries. ${ }^{4}$

Age matched controls were recruited accordingly. Controls were women with normal menstrual cycles, having no signs of clinical hyperandrogenism and no polycystic ovaries on ultrasonography. Socio economic status is being classified for both cases and controls on modified Kuppuswamy scale. ${ }^{6}$

All subjects were then tested for pregnancy, abnormal thyroid and prolactin levels. Subjects having abnormal prolactin or thyroid levels, or positive pregnancy test were excluded from the study.

All subjects were tested for metabolic abnormalities.

\section{Measurement of parameters of metabolic syndrome}

- Waist circumference was calculated between the highest point of iliac crest and costal margin

- Blood pressure was measured from standard sphygmomanometer

- Lipid levels was estimated by commercially available enzymatic in vitro assay kits and expressed as $\mathrm{mg} / \mathrm{dl}$

- Blood Sugars (fasting and glucose tolerance) were measured by glucose oxidase-peroxidase method in the biochemistry department laboratory of study hospital.

- All subjects (cases and controls) underwent Oral Glucose Tolerance Test (OGTT)

- 2-hour glucose levels were measured after 75 gm glucose intake.

\section{Statistical analysis}

Statistical testing was conducted with the statistical package version SPSS 17.0. Continuous variables are presented as mean $\pm \mathrm{SD}$, and categorical variables are presented as absolute numbers and percentage. The comparison of normally distributed continuous variables between the groups was performed using Student's t test. Nominal categorical data between the groups were compared using Chi-squared test or Fisher's exact test as appropriate. An odds ratio with $95 \%$ CI was calculated as the ratio of the odds of the outcome in the patients using Logistic regression analysis. $\mathrm{p}<0.05$ was considered statistically significant.

\section{RESULTS}

Ages of cases and controls were comparable. In the study the mean age of PCOS cases was 19.5 years and $66 \%$ of 
cases were under 20 years of age. None of the cases were aged more than 30 years. Maximum cases of PCOS (64\%) identified belong to upper middle class on modified Kuppuswamy scale. Majority of the cases reported in the study had oligomenorhhea as their chief complaint. Of the 50 cases 42 had oligomenorrhea. Weight and BMI was significantly higher among PCOS cases than controls. $50 \%$ of PCOS cases were with weight $>60 \mathrm{kgs}$ (Table 1$)$.
Mean weight among cases was $63.30 \pm 10.24 \mathrm{~kg}$. BMI was statistically different among cases and controls with an odds ratio of 1.179. ( $\mathrm{p}=0.014)$ (Table 1). Mean waist was $93.24 \pm 11.66 \mathrm{~cm}$ in cases to $85.10 \pm 8.23$ was found to be statistically significantly different among cases and controls ( $p$ value $<0.001$ ). The odds of having PCOS if waist circumference is $>88 \mathrm{~cm}$ was found to be 1.08 times the control (Table 2).

Table 1: BMI differences among cases and controls.

\begin{tabular}{|lllllll|} 
& Control & & Cases & & p value & Odds ratio (95\% CI) \\
\hline Height & Mean \pm SD & Min-Max & Mean \pm SD & Min-Max & & \\
\hline Weight & $153.60 \pm 5.04$ & $145-165$ & $154.46 \pm 5.83$ & $142-164$ & 0.428 & $1.030(0.957-1.108)$ \\
\hline BMI & $58.26 \pm 6.24$ & $48-70$ & $63.30 \pm 10.24$ & $46-90$ & 0.006 & $1.077(1.021-1.136)$ \\
\hline
\end{tabular}

Table 2: Waist hip ratio differences among control and cases.

\begin{tabular}{|lllllll|} 
& Control & \multicolumn{3}{c}{ Cases } \\
& Mean \pm SD & Min-Max & Mean \pm SD & Min-Max & p value & Odds ratio (95\% CI) \\
\hline Waist & $85.10 \pm 8.23$ & $72-104$ & $93.24 \pm 11.66$ & $68-116$ & $<0.001$ & $1.084(1.036-1.133)$ \\
\hline Hip & $92.48 \pm 7.30$ & $80-108$ & $101.72 \pm 8.49$ & $84-124$ & $<0.001$ & $1.150(1.084-1.221)$ \\
\hline Waist-hip ratio & $0.92 \pm 0.03$ & $0.8-1.0$ & $0.92 \pm 0.06$ & $0.8-1.0$ & 0.634 & $0.141(0.000-450.19)$ \\
\hline
\end{tabular}

Table 3: Lipid profile and OGTT among cases and controls.

\begin{tabular}{|llllllll|} 
& Control & & Cases & & Min-Max & p value & Odds ratio (95\% CI) \\
& Mean \pm SD & Min-Max & Mean \pm SD & Min & & $0.999(0.980-1.018)$ \\
\hline Triglyceride levels & $120.96 \pm 14.30$ & $92-154$ & $124.50 \pm 25.07$ & $88-172$ & 0.909 & $0.1230(1.120-1.352)$ \\
\hline HDL & $35.14 \pm 4.13$ & $29-43$ & $41.74 \pm 7.39$ & $25-65$ & $<0.001$ & $1.2307(1.051-1.145)$ \\
\hline Fasting blood glucose & $79.12 \pm 10.77$ & $65-104$ & $92.28 \pm 14.24$ & $68-139$ & $<0.001$ & 1.097 \\
\hline $\begin{array}{l}\text { Oral glucose } \\
\text { tolerance test }\end{array}$ & $101.12 \pm 12.60$ & $84-142$ & $123.28 \pm 23.31$ & $94-189$ & $<0.001$ & $1.084(1.045-1.124)$ \\
\hline
\end{tabular}

Table 4: Frequency of metabolic syndrome (MS).

\begin{tabular}{|c|c|c|c|c|c|c|}
\hline \multirow{2}{*}{$\begin{array}{l}\text { Metabolic } \\
\text { syndrome }\end{array}$} & \multicolumn{2}{|l|}{ Control } & \multicolumn{2}{|l|}{ Cases } & \multirow[b]{2}{*}{$p$ value } & \multirow{2}{*}{ Odds ratio $(95 \% \mathrm{CI})$} \\
\hline & Frequency & Percentage & Frequency & Percentage & & \\
\hline Yes & 4 & $8 \%$ & 17 & $34 \%$ & \multirow{3}{*}{0.003} & \multirow{3}{*}{$5.92(1.825-19.231)$} \\
\hline No & 46 & $92 \%$ & 33 & $66 \%$ & & \\
\hline Total & 50 & $100 \%$ & 50 & $100 \%$ & & \\
\hline
\end{tabular}

Table 5: Independent predictors of PCOS using multivariate regression analysis.

\begin{tabular}{|lllllll|}
\hline & B & Standard error & Significance & Odds ratio of exposure (B) & 95\% CI \\
\hline BMI & -0.422 & 0.159 & 0.008 & 1.525 & 1.117 & 2.084 \\
\hline Hip circumference & 0.253 & 0.074 & 0.001 & 1.288 & 1.115 & 1.487 \\
\hline OGTT & 0.075 & 0.029 & 0.009 & 1.078 & 1.019 & 1.140 \\
\hline
\end{tabular}

Hip circumference was also statistically different but waist-hip ratio was not found to be different among cases and controls ( $\mathrm{p}$-value $=0.634$ ) (Table 2). Clinical hyperandrogenism including hirsutism and other changes 
like acne was found in 32 of 50 cases. Disturbing acne was found in 9 of the 32 cases with signs of clinical hyperandrogenism.

Means of HDL, fasting blood glucose and oral glucose tolerance test were found to be statistically different among PCOS cases and controls (Table 3).

In this study 17 of the 50 cases and 4 of the controls met the criteria for metabolic syndrome. Metabolic syndrome was statistically significantly associated with PCOS. The odds of having metabolic syndrome were found to be 5.92 with a $95 \%$ confidence interval of 1.825-19.231 (Table 4).

Multivariate logistic regression analysis (step-wise method) showed that BMI, Hip circumference and Oral Glucose Tolerance Test (OGTT) were independent and significant predictors of polycystic ovarian syndrome.

Table 5 shows odds-ratio for BMI as an independent predictor was 1.525 (95\% C.I. 1.117-2.084), odds-ratio for Hip circumference as an independent predictor was 1.288 (95\% C.I. 1.115-1.487), odds-ratio for impaired oral glucose tolerance test as an independent predictor was 1.078 (95\% C.I. 1.019-1.140).

\section{DISCUSSION}

Mean weight was $63.30 \pm 10.24$ in the cases whereas it was 58.26 \pm 6.24 in control group (p-value $=0.004)$. Mean BMI of the cases was $26.52 \pm 3.99$ whereas it was $24.72 \pm 2.70$ for the controls (p-value $=0.009$ ). BMI of $>25 \mathrm{~kg} / \mathrm{m}^{2}$ was found in $54 \%$ of cases as compared with $38 \%$ of controls. Mean BMI in a study by Knochenhauer et al in United States was $36.5 \pm 9.1 \mathrm{~kg} / \mathrm{m}^{2} .{ }^{7}$ However in India mean BMI in a study by Shivaprakash et al was $27.8 \pm 2.76$. $^{8}$ This can be attributed to difference in body habitus among different ethnic groups.

Abdominal obesity as measured by waist circumference > $88 \mathrm{~cm}$ was found to be significantly associated with PCOS cases. Mean value in cases was 93.24 \pm 11.66 and in controls was $85.10 \pm 8.23$ (p-value $<0.001$ ). Measurement at the hip was also significantly different in cases and controls. Mean hip circumference was $101.72 \pm 8.49$ in controls and $92.48 \pm 7.30$ in cases (p-value <0.001). However, waist-hip ratio was not found to be significantly different between cases and controls. All the diagnostic criteria of metabolic syndrome include waist circumference only and not waist-hip ratio. This is different when compared to a similar study done by Kalra et al in South India which showed that waist- hip ratio was a better predictor of incidence of metabolic syndrome and PCOS than each individual component. ${ }^{9}$ Perhaps, there are some differences in the body habitus of the two populations that needs to be studied further.

Using multi-variate regression analysis, BMI and 2-hour Oral Glucose Tolerance Test were found to be independent predictors of polycystic ovarian syndrome, odds ratio of 1.525 (95\% C.I 1.019-1.140). According to Rong $\mathrm{Li}$ et al also, BMI is an independent predictor of PCOS with Odds ratio of 1.420 (95\% CI: 1.328-1.518). ${ }^{10}$

In this study, metabolic syndrome was found in 17 out of the 50 cases of PCOS (34\%) and only 4 out of the 50 controls $(8 \%)$. This is similar to a study by Jamil et al which identified Metabolic syndrome in 112 (42.6\%) of the PCOS women and $45(17.1 \%)$ of the control $(\mathrm{p}<0.001) .{ }^{11}$ Odds ratio of having metabolic syndrome in PCOS patients in my study was 5.92 with a $95 \%$ CI of (1.825-19.231). This statistically significant difference ( $\mathrm{p}$-value $=0.003$ ) shows that there is a clear association between the metabolic syndrome and PCOS.

The relationship between PCOS and metabolic syndrome (MS) is possibly mutual. Thus, not only MS is prevalent among PCOS women, but also women with MS may commonly present the reproductive/endocrine hallmarks of PCOS.

Strengths of the study include the baseline characteristics were adequately matched for the cases and controls. As only one person was collecting the data, inter-observer variation was minimized. The study was not blinded therefore subconscious bias while evaluating the patients cannot be excluded.

Only patients coming to the outpatient clinic in the hospital were included. This leads to Berksonian bias. This study was conducted in a single center in the North East of India. Sample size was limited due to the number of cases that were recruited from the outpatient department over a short period of one and a half years.

\section{CONCLUSION}

Association between PCOS and metabolic syndrome is statistically significant. As attention is focused on the emerging epidemic of type 2 diabetes and obesity, more energy is being directed toward earlier detection, improved therapies, and potential prevention. Interestingly, many of the features of the metabolic syndrome, including insulin resistance, obesity, and dyslipidaemias, are also present in PCOS. Is PCOS an early manifestation of metabolic syndrome?

PCOS and metabolic syndrome are two entities that are intrinsically linked to each other and the early identification of one may lead to the diagnosis and management of the other. With the growing burden of lifestyle diseases in our society, it is imperative to further study the intricate pathophysiological mechanisms in order to completely understand the association of polycystic ovarian syndrome and metabolic syndrome.

Further studies on a larger scale with multi-centric analysis and double blinding are needed to validate the results of this study. 


\section{ACKNOWLEDGMENTS}

Authors would like to thank to faculties and post graduate students for their help.

Funding: No funding sources

Conflict of interest: None declared

Ethical approval: The study was approved by the Institutional Ethics Committee

\section{REFERENCES}

1. Ehrmann DA. Polycystic ovary syndrome. N Engl J Med. 2005;352(12):1223-36.

2. Norman RJ, Mahabeer S, Masters S. Ethnic differences in insulin and glucose response to glucose between white and Indian women with polycystic ovary syndrome. Fertil Steril. 1995;63(1):58-62.

3. Apridonidze T, Essah PA, Iuorno MJ, Nestler JE. Prevalence and characteristics of the metabolic syndrome in women with polycystic ovary syndrome. J Clin Endocrinol Metab. 2005;90:192935 .

4. The Rotterdam ESHRE/ASRM-sponsored PCOS consensus workshop group. Revised 2003 consensus on diagnostic and long-term health risks related to polycystic ovary syndrome (PCOS). Human Reprod. 2004;19:41-7.

5. Coskun A, Ercan O, Arikan DC, Özer A, Kilinc M, Kiran G, et al. Modified Ferriman-Gallwey hirsutism score and androgen levels in Turkish women. Eur J Obstel Gynecol Reprod Biol. 2011;154(2):167-71.
6. Sharma R. Kuppuswamy's socioeconomic status scale - revision for 2011 and formula for real-time updating. Indian J Pediatr. 2012;79(7):961-2.

7. Knochenhauer ES, Key TJ, Kahsar-Miller M, Waggoner W, Boots LR, Azziz R. Prevalence of the polycystic ovary syndrome in unselected black and white women of the southeastern United States: a prospective study. J Clin Endocrinol Metab. 1998;83(9):3078-82.

8. Shivaprakash G, Basu A, Kamath A. Acanthosis Nigricans in PCOS patients and its relation with type 2 diabetes mellitus and body mass at a tertiary care hospital in Southern India. J Clin Diagn Res. 2013;7(2):317-9.

9. Kalra A, Nair S, Rai L. Association of obesity and insulin resistance with dyslipidemia in Indian women with polycystic ovarian syndrome. Indian J Med Sci. 2006;60:447-53.

10. Li R, Yu G, Yang D. Prevalence and predictors of metabolic abnormalities in Chinese women with PCOS: a cross- sectional study. BMC Endocr Disord. 2014; $14: 76$.

11. Jamil AS, Alalaf SK, Al-tawil NG, Al-shawaf T. A case-control observational study of insulin resistance and metabolic syndrome among the four phenotypes of polycystic ovary syndrome based on Rotterdam criteria. Reprod Health. 2015;12:7.

Cite this article as: Batra I, Baidya JL, Pradhan M. Association of polycystic ovarian syndrome and metabolic syndrome in Tripura, India. Int J Reprod Contracept Obstet Gynecol 2020;9:2452-6. 